\title{
The prognostic value of cardiopulmonary exercise testing in interstitial lung disease: a systematic review
}

\author{
Shaney L. Barratt (10), Richard Davis ${ }^{1}$, Charles Sharp (i) $^{3}$ and \\ John D. Pauling ${ }^{4,5}$
}

Affiliations: ${ }^{1}$ Academic Respiratory Unit, School of Clinical Sciences, University of Bristol, Bristol, UK. ${ }^{2}$ Bristol Interstitial Lung Disease Service, North Bristol NHS Trust, Westbury-on-Trym, Bristol, UK. ${ }^{3}$ Gloucestershire Hospitals NHS Foundation Trust, Gloucester, UK. ${ }^{4}$ Department of Pharmacy and Pharmacology, University of Bath, Bath, UK. ${ }^{5}$ Royal National Hospital for Rheumatic Diseases, Royal United Hospitals NHS Foundation Trust, Bath, UK.

Correspondence: Shaney L. Barratt, Consultant Respiratory Physician and Honorary Associate Researcher, North Bristol NHS Trust, Southmead Hospital, Westbury-on-Trym, Bristol BS10 5NB, UK. E-mail: shaney. barrattanbt.nhs.uk

ABSTRACT The heterogeneity of interstitial lung disease (ILD) results in prognostic uncertainty concerning end-of-life discussions and optimal timing for transplantation. Effective prognostic markers and prediction models are needed. Cardiopulmonary exercise testing (CPET) provides a comprehensive assessment of the physiological changes in the respiratory, cardiovascular and musculoskeletal systems in a controlled laboratory environment. It has shown promise as a prognostic factor for other chronic respiratory conditions. We sought to evaluate the prognostic value of CPET in predicting outcomes in longitudinal studies of ILD.

MEDLINE, Embase and the Cochrane Database of Systematic Reviews were used to identify studies reporting the prognostic value of CPET in predicting outcomes in longitudinal studies of ILD. Study quality was assessed using the Quality in Prognosis Study risk of bias tool.

Thirteen studies were included that reported the prognostic value of CPET in ILD. All studies reported at least one CPET parameter predicting clinical outcomes in ILD, with survival being the principal outcome assessed. Maximum oxygen consumption, reduced ventilatory efficiency and exercise-induced hypoxaemia were all reported to have prognostic value in ILD. Issues with study design (primarily due to inherent problems of retrospective studies, patient selection and presentation of numerous CPET parameters), insufficient adjustment for important confounders and inadequate statistical analyses limit the strength of the conclusions that can be drawn at this stage.

There is insufficient evidence to confirm the value of CPET in facilitating "real-world" clinical decisions in ILD. Additional prospective studies are required to validate the putative prognostic associations reported in previous studies in carefully phenotyped patient populations.

@ERSpublications

There is presently insufficient evidence to confirm the value of CPET in facilitating "real-world" clinical decisions in ILD. Additional prospective studies are required to validate the putative prognostic associations reported in previous studies. https://bit.ly/3dfp5kq

Cite this article as: Barratt SL, Davis R, Sharp C, et al. The prognostic value of cardiopulmonary exercise testing in interstitial lung disease: a systematic review. ERJ Open Res 2020; 6: 00027-2020 [https://doi.org/10.1183/23120541.00027-2020].

This article has supplementary material available from openres.ersjournals.com

Received: 18 Jan 2020 | Accepted after revision: 22 April 2020

Copyright $\odot$ ERS 2020. This article is open access and distributed under the terms of the Creative Commons Attribution Non-Commercial Licence 4.0. 


\section{Introduction}

The heterogeneity of interstitial lung disease (ILD) $[1,2]$ presents challenges for patients and clinicians in terms of treatment choices, optimal timing of end-of-life discussions [3], or referral for transplantation [4] and clinical trial design $[5,6]$.

Cardiopulmonary exercise testing (CPET) provides a comprehensive assessment of the physiological changes that occur in the respiratory, cardiovascular and musculoskeletal systems during exercise, in a controlled laboratory environment $[7,8]$, and is considered the gold standard for evaluating maximal/symptom-limited exercise tolerance in patients with pulmonary and cardiac disease [9]. Although CPET has been available for decades, recent evidence is emerging to support its use in the prognostication of chronic cardiopulmonary disease $[10,11]$, with increasing interest in its application in ILD [12].

Maximum/peak oxygen consumption $\left(\mathrm{VO}_{2 \max }\right.$ or peak $\left.\mathrm{VO}_{2}\right)$ is a measurement of the capacity for aerobic exercise and is determined by variables that define oxygen delivery by the Fick equation [13]. In patients with ILD, limitations on exercise may be the consequence of either ventilatory mechanical limitation (by reaching their ventilatory ceiling, typically thought to be $80 \%$ of maximal voluntary ventilation (MVV)), abnormal gas exchange (or reduction in ventilatory efficiency; indicated by variables such as the increment in minute ventilation (VE) relative to carbon dioxide production $\left(\mathrm{VE} / \mathrm{VCO}_{2}\right)$ ) and/or diffusion limitation (indicated by variables such as reduction in oxygenation $>5 \%$ or hypoxia at anaerobic threshold (AT)/peak exercise) [13].

The primary objective of this systematic literature review was to evaluate the prognostic value of CPET in predicting disease-specific outcomes in longitudinal studies of ILD. If a prognostic role for CPET were confirmed, it could be used to guide earlier intervention for at-risk patients and support cohort enrichment for ILD clinical trials.

\section{Materials and methods}

The study protocol was prepared in accordance with Preferred Reporting Items for Systematic Reviews and Meta-Analyses (PRISMA) guidelines [14] and registered in the International Prospective Register of Systematic Reviews (PROSPERO 110198/2018) (study commencement date 1 November 2018, completion date 30 September 2019). In brief, eligible studies included cohort (retrospective or prospective) studies reporting the prognostic value of CPET results in adult populations of ILD.

The primary objective was to evaluate the prognostic value of CPET in predicting disease course and outcomes in longitudinal studies of ILD. We explored the relationship between CPET and a broad range of relevant clinical outcomes including, but not limited to, relevant disease outcomes (e.g. death, hospitalisation), potential surrogates of disease severity (e.g. worsening lung physiology, etc.), and future deterioration in health-related quality of life (HRQoL) and/or functional status. Where possible, a comparison of the prognostic value of CPET was made across different ILD subtypes.

Studies were excluded if an ILD cohort was not described and reported separately. Non-original research publications and abbreviated reports were excluded. Randomised controlled trials (RCTs) were excluded as we did not expect this to be an appropriate methodological design for assessing the prognostic value of CPET. An amendment to our originally registered protocol (English language articles only) enabled the inclusion of a relevant non-English (French) publication.

The search criteria were developed in accordance with search recommendations for systematic reviews of evaluations of prognostic variables [15]. Electronic searches were performed in MEDLINE, Embase and the Cochrane Database of Systematic Reviews (CDSR), with no publication date or language restrictions. Full details of the specific search criteria applied are presented as supplementary material 1 . All titles and abstracts were screened independently by two review authors (RD and CS), and agreement was assessed using Cohen's kappa statistics [16]. Any discrepancies/disagreements were resolved by discussion between reviewers and included a third party (SLB) if necessary.

A formal systematic review management platform was not used for this study. EndNote (Alfasoft Limited) was used to facilitate the combination of multiple database results and deduplication. A standardised data extraction form was used (independently by RS and CS, with subsequent verification by SLB) to extract relevant study details from selected studies.

A meta-analysis was planned if appropriate and feasible. A narrative, qualitative data synthesis was planned if wide heterogeneity in study design and CPET analysis precluded quantitative analysis. Study quality was assessed using the QUIPS (Quality in Prognosis Study) risk of bias tool by two reviewers (RD and CS) [17], with agreement measured using Cohen's kappa (supplementary material 2). 


\section{Results}

Simultaneous searches of Embase $(n=573)$ and MEDLINE $(n=373)$, performed on 13 April 2019, identified 946 articles. A search of the CDSR did not identify any additional studies. As anticipated, we did not identify any relevant RCTs in our study selection process and no studies were excluded on the basis of an RCT design. After removal of duplicates, 658 titles and abstracts were screened for eligibility. There was moderate initial agreement between the two reviewers (Cohen's kappa 0.462, supplementary material 3). Discordance for 20 studies was due to a single non-clinically trained reviewer choosing to include questionable studies for consideration (all of which were easily resolved through discussion and subsequently excluded). Due to the nature of discordance, it was not felt that retraining reviewers and formally repeating the title and abstract selection process would benefit the review process.

Following full text review, 13 studies were eligible for full data extraction (figure 1).

\section{Study design}

Table 1 summarises the study design and reported findings of the final 13 studies.

The majority were retrospective cohort analyses (11/13, 85\%), with variable follow-up (the majority $<4$ years [7, 18-25], one study with 5 year follow-up [26]; range 23 days-20 years follow-up [19, 27]).

There were two prospective studies $[28,29]$. One investigated the relationship between CPET and survival characteristics in IPF with variable follow-up between 9 and 64 months [28]. The other used CPET as part of a wider investigation into the role of exercise testing in the prognostication of ILD and followed patients for a fixed period of 40 months [29].

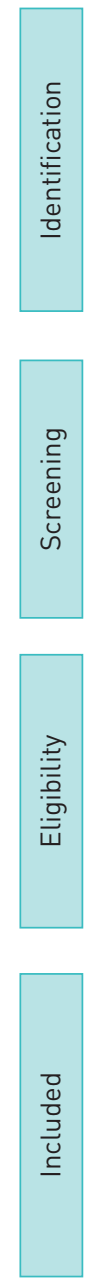

Articles identified through MEDLINE search, $n=373$
Articles identified through Embase search, $n=573$
Articles identified through CDSR search, $n=0$

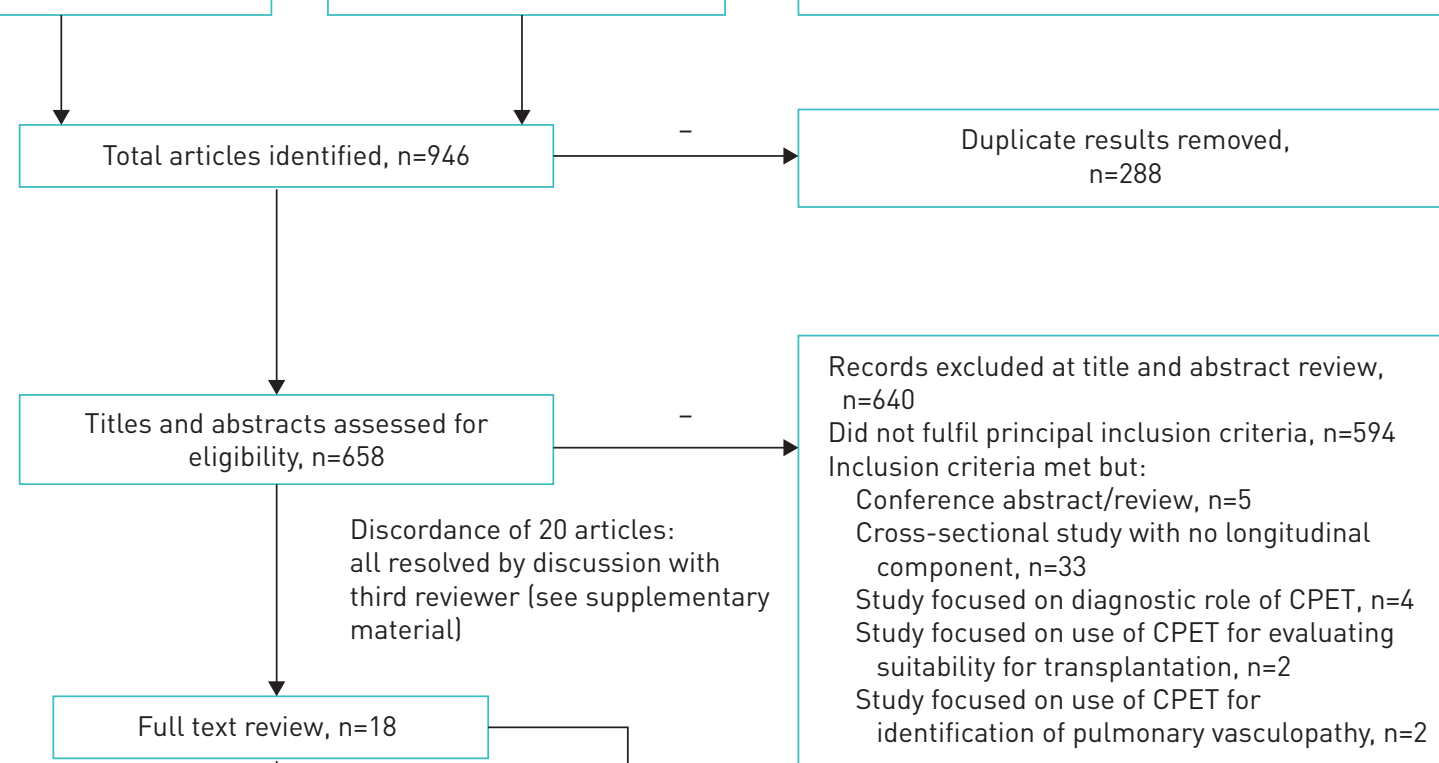

Studies included in qualitative synthesis, $n=13$

Full text articles excluded, $n=5$ lcross-sectional study of CPET with no longitudinal component, $\mathrm{n}=5$

FIGURE 1 Study selection flow diagram presented according to PRISMA statement. CDSR: Cochrane Database of Systematic Reviews; CPET: cardiopulmonary exercise testing. 
TABLE 1 Study characteristics of papers selected for full data extraction

\begin{tabular}{|c|c|c|c|c|c|c|c|c|}
\hline $\begin{array}{l}\text { First author, } \\
\text { date [ref], } \\
\text { origin }\end{array}$ & Description & $\begin{array}{l}\text { Study population and } \\
\text { attrition }\end{array}$ & $\begin{array}{l}\text { CPET method and } \\
\text { CPET parameters }\end{array}$ & Exclusion & Disease outcomes & $\begin{array}{l}\text { Statistical methods to } \\
\text { investigate CPET and } \\
\text { outcome }\end{array}$ & $\begin{array}{l}\text { Summary of key reported } \\
\text { outcomes }\end{array}$ & Comments \\
\hline $\begin{array}{l}\text { TRIANTAFILLIDOU } \\
2013 \text { [28], } \\
\text { Greece }\end{array}$ & $\begin{array}{l}\text { Prospective study } \\
\text { evaluating prognostic } \\
\text { role of 6MWT and } \\
\text { CPET in IPF. } \\
\text { Follow-up } \\
\text { 9-64 months. }\end{array}$ & 25 pts with IPF & $\begin{array}{l}\text { Cycle ergometer, } \\
\text { pulse oximetry. } \\
\mathrm{VE} / \mathrm{VCO}_{2} \text { slope, } \\
\mathrm{VO}_{2} \text { peak } / \mathrm{kg} \text {, } \\
\mathrm{VE} / \mathrm{VCO}_{2} \text { ratio } \\
\text { at AT. }\end{array}$ & $\begin{array}{l}\text { Significant PH (PASP } \\
>45 \mathrm{mmHg} \text { on ECHO), pts } \\
\text { taking beta blockers. } \\
\text { Pulmonary fibrosis due to } \\
\text { environmental and } \\
\text { occupational exposure, } \\
\text { drug toxicity or } \\
\text { autoimmune } \\
\text { rheumatological disease. }\end{array}$ & Survival & $\begin{array}{l}\text { Parameters of study were } \\
\text { evaluated by Wald test, } \\
\text { likelihood ratio test and the } \\
\text { score (log-rank) tests with } \\
\text { Bonferroni correction. } \\
\text { Parameters achieving } \\
\text { statistical significance } \\
\text { were then evaluated in a } \\
\text { multiple regression Cox } \\
\text { proportional hazard model } \\
\text { with a stepwise model } \\
\text { selection. }\end{array}$ & 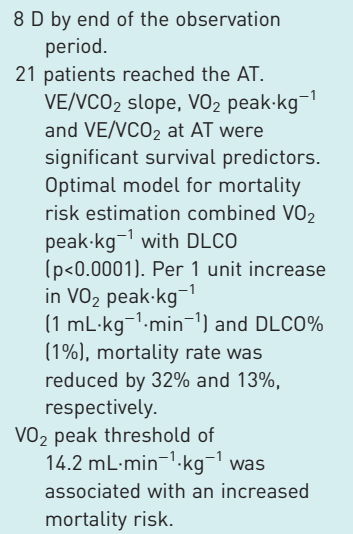 & $\begin{array}{l}\text { Prospective study with } \\
\text { low mortality rate } \\
\text { in small numbers } \\
\text { of pts. } \\
\text { Data generated from } \\
\text { sub-analysis of } \\
\text { RCT. }\end{array}$ \\
\hline $\begin{array}{l}\text { VAINSHELBoIM } \\
2016 \text { [29], } \\
\text { Israel }\end{array}$ & $\begin{array}{l}\text { Prospective } \\
\text { observational study } \\
\text { evaluating role of } \\
12 \text { week exercise } \\
\text { training programme } \\
\text { on survival at } \\
40 \text { months follow-up. } \\
\text { Evaluation of the role } \\
\text { of CPET variables in } \\
\text { the prognostication } \\
\text { of IPF. }\end{array}$ & 34 pts with IPF & $\begin{array}{l}\text { Cycle ergometer, } \\
\text { pulse oximetry. } \\
\text { Peak VO } \mathrm{V}_{2} \cdot \mathrm{kg}^{-1} \text {, } \\
\text { peak work rate, } \\
{\mathrm{VE} / \mathrm{NO}_{2} \text { nadir, }}_{\mathrm{VE} / \mathrm{NCO}_{2} \text { ratio at }} \\
\text { AT, tidal volume } \\
\text { reserve. }\end{array}$ & $\begin{array}{l}\text { Non-IPF ILD. Clinically } \\
\text { unstable in preceding } \\
3-6 \text { months, severe } \\
\text { comorbid illness, unstable } \\
\text { cardiac disease and any } \\
\text { orthopaedic or } \\
\text { neurological } \\
\text { contraindications to CPET. }\end{array}$ & $\begin{array}{l}\text { Mortality or } \\
\text { transplantation }\end{array}$ & $\begin{array}{l}\text { ROC curve analysis was used } \\
\text { to determine cut-off points } \\
\text { of CPET variables for } \\
\text { mortality. Cox regression } \\
\text { analysis for survival } \\
\text { analysis and comparison } \\
\text { between significant cut-off } \\
\text { points (log-rank test). HR } \\
\text { for death or LTx (Wald } \\
\text { test). }\end{array}$ & 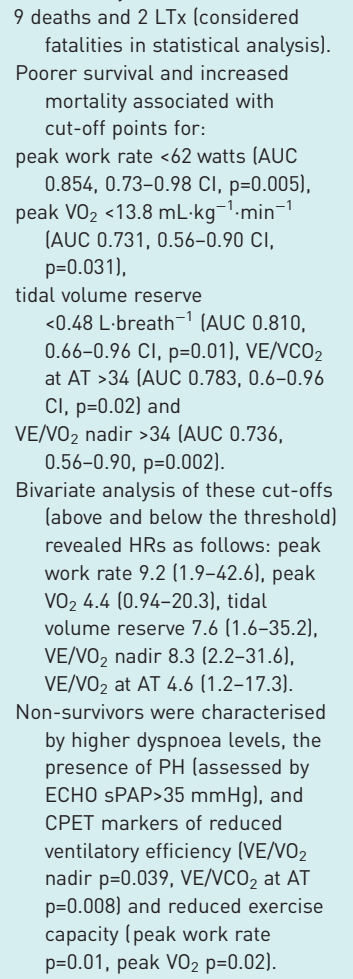 & $\begin{array}{l}\text { Prospective } \\
\text { observational study } \\
\text { analysis as part of } \\
\text { a wider } \\
\text { single-centre RCT. } \\
\text { Underpowered to } \\
\text { detect survival } \\
\text { differences } \\
\text { between groups. } \\
\text { Small sample size. } \\
\text { Higher prevalence of } \\
\text { PH in } \\
\text { non-survivors. }\end{array}$ \\
\hline
\end{tabular}




\begin{tabular}{|c|c|c|c|c|c|c|c|c|}
\hline $\begin{array}{l}\text { First author, } \\
\text { date [ref], } \\
\text { origin }\end{array}$ & Description & $\begin{array}{l}\text { Study population and } \\
\text { attrition }\end{array}$ & $\begin{array}{l}\text { CPET method and } \\
\text { CPET parameters }\end{array}$ & Exclusion & Disease outcomes & $\begin{array}{l}\text { Statistical methods to } \\
\text { investigate CPET and } \\
\text { outcome }\end{array}$ & $\begin{array}{l}\text { Summary of key reported } \\
\text { outcomes }\end{array}$ & Comments \\
\hline $\begin{array}{l}\text { KING } 2001 \text { [23], } \\
\text { USA }\end{array}$ & $\begin{array}{l}\text { Retrospective analysis } \\
\text { of clinical, } \\
\text { radiological and } \\
\text { physiological } \\
\text { parameters } \\
\text { predicting survival in } \\
\text { IPF. Median } \\
\text { follow-up } 20 \text { months } \\
\text { (maximum } \\
14.8 \text { years). }\end{array}$ & $\begin{array}{l}238 \text { IPF pts with } \\
\text { histological UIP. } \\
80 \text { pts excluded from } \\
\text { the final model } \\
\text { derivation. }\end{array}$ & $\begin{array}{l}\text { Cycle ergometer, } \\
\text { blood gas } \\
\text { analysis. } \\
\mathrm{P}^{(\mathrm{A}-\mathrm{a}) \mathrm{O}_{2}} \\
\text { corrected for } \mathrm{FiO}_{2}, \\
\mathrm{VD}_{2} / \mathrm{NT}, \mathrm{VO}_{2} \text {, } \\
\text { maximal } \\
\text { workload. }\end{array}$ & $\begin{array}{l}\text { CTD, left ventricular failure, } \\
\text { occupational and } \\
\text { environmental exposure, } \\
\text { or history of drug } \\
\text { exposure known to cause } \\
\text { pulmonary fibrosis. } \\
\text { Incomplete case records. }\end{array}$ & $\begin{array}{l}\text { Survival (defined as death } \\
\text { or time of censoring: } \\
\text { censored if still alive } \\
\text { at last contact } n=79 \text {, } \\
\text { received single } L T x \\
n=11 \text {, double } L T x n=1 \text {, } \\
\text { or heart and } L T x n=1 \\
\text { or e) died from other } \\
\text { cause than IPF (n=12). }\end{array}$ & $\begin{array}{l}\text { Kaplan-Meier survival curves } \\
\text { developed for group, } \\
\text { stratified by sex, age and } \\
\text { smoking status. Univariate } \\
\text { Cox proportional hazards } \\
\text { regression analysis } \\
\text { ladjusted for age and } \\
\text { smokingl for each variable. } \\
\text { Variables with p<0.25 } \\
\text { included in multivariate } \\
\text { analysis. Pearson's } \\
\text { correlation to avoid } \\
\text { multicollinearity. Forward } \\
\text { elimination process used } \\
\text { to develop preliminary } \\
\text { model. Multivariable } \\
\text { influential points removed. } \\
\text { Composite scoring system } \\
\text { developed, weighting } \\
\text { categories according to p } \\
\text { values and HR, and using } \\
\text { Akaike's information } \\
\text { criteria. }\end{array}$ & $\begin{array}{l}155 \mathrm{D} \text { (125 IPF, } 19 \text { other causes, } 11 \\
\text { unknown and attributed to IPF). } \\
105 \text { patients censored (n=79 alive } \\
\text { at time of analysis, } n=13 \mathrm{LTx}, \\
\mathrm{n}=12 \text { non-IPF deaths, } \mathrm{n}=1 \text { lost } \\
\text { to follow-up). } \\
\text { Composite scoring model } \\
\text { developed to predict survival in } \\
\text { IPF lincluded age, smoking } \\
\text { history, clubbing, extent of } \\
\text { profusion of interstitial } \\
\text { opacities, presence/absence of } \\
\mathrm{PH} \text { on } \mathrm{CXR}, \% \text { predicted TLC } \\
\text { and } \mathrm{PaO}_{2} \text { at the end of maximal } \\
\text { exercise). } \\
\text { Exercise } \mathrm{PaO} \mathrm{O}_{2} \text { only exercise } \\
\text { variable included in the model, } \\
\text { accounting for } 10.5 \% \text { of score } \\
\text { (PaO } \mathrm{O}_{2} \text { maximal exercise } \mathrm{HR} \\
0.74, \mathrm{Cl} 0.67-0.82, \mathrm{p}<0.0001) .\end{array}$ & $\begin{array}{l}\text { CPET performed in } \\
\text { study as part of } \\
\text { wider analysis of } \\
\text { predictive factors in } \\
\text { IPF. } \\
\text { Histological UIP } \\
\text { increased potential } \\
\text { selection bias of a } \\
\text { less severe IPF } \\
\text { population. } \\
\text { The radiological } \\
\text { component used } \\
\text { CXR rather than } \\
\text { HRCT in early } \\
\text { years of the study. } \\
\text { Only } 158 / 238 \text { l66\%) of } \\
\text { the original cohort } \\
\text { were used to derive } \\
\text { the complete } \\
\text { model and thus } \\
\text { possibility for } \\
\text { selection bias. }\end{array}$ \\
\hline $\begin{array}{l}\text { Мıк' } 2003 \text { [21], } \\
\text { Japan }\end{array}$ & $\begin{array}{l}\text { Retrospective study: } \\
\text { evaluation of the } \\
\text { predictive value of } \\
\text { CPET for IPF } \\
\text { respiratory deaths. } \\
\text { Mean follow-up } \\
2.7 \text { years } \\
\text { (7.2 months- } \\
9.0 \text { years). }\end{array}$ & 41 IPF pts. & $\begin{array}{l}\text { Exercise treadmill } \\
\text { (Sheffield } \\
\text { protocoll). } \mathrm{PaO}{ }_{2,} \\
\mathrm{PaCO}_{2}, \mathrm{HR}, \\
\text { respiratory } \\
\text { frequency (f), } \mathrm{Vt}, \\
\mathrm{VE}, \mathrm{peak} \mathrm{VO}_{2}, \\
\mathrm{VE} / \mathrm{VO}_{2}, \mathrm{VE} / \mathrm{VCO}_{2}, \\
\mathrm{VO}_{2} / \mathrm{HR}_{1} \mathrm{AaO}_{2} \\
\text { and } \mathrm{PaO}_{2} \text { slope. }\end{array}$ & $\begin{array}{l}\text { CTD, sarcoid, OP, EP, HP, } \\
\text { cardiac disease, anaemia, } \\
\text { primary cardiac disease, } \\
\text { PVD, cancer, pleural/chest } \\
\text { wall disorders including } \\
\text { respiratory muscle } \\
\text { weakness. Steroid or } \\
\text { immunosuppressive } \\
\text { treatment prior to study } \\
\text { entry. Death from a } \\
\text { non-respiratory cause } \\
\text { during follow-up. }\end{array}$ & Respiratory death & $\begin{array}{l}\text { Exercise parameters (between } \\
\text { groups split by } \mathrm{PaO}_{2} \text { slope) } \\
\text { compared using } \\
\text { Mann-Whitney. Univariate } \\
\text { Cox proportional hazards } \\
\text { model to compare initial } \\
\text { parameters then entered } \\
\text { into multiple regression } \\
\text { analysis using stepwise } \\
\text { evaluation. Relationship } \\
\text { between } \mathrm{PaO}_{2} \text { slope and } \\
\text { other variables were } \\
\text { analysed by linear } \\
\text { regression with stepwise } \\
\text { technique. Survival times } \\
\text { compared using } \\
\text { Kaplan-Meier curves and } \\
\text { statistical significance } \\
\text { determined by log-rank } \\
\text { test. }\end{array}$ & 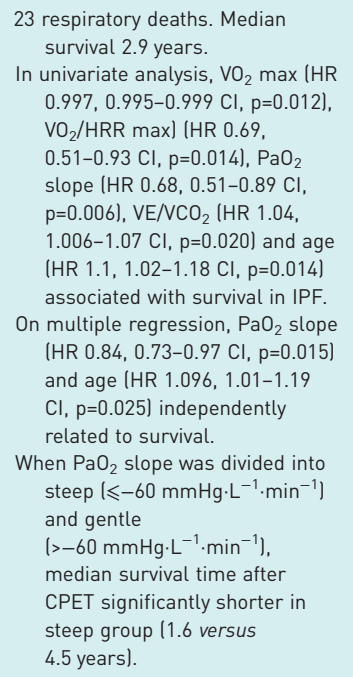 & $\begin{array}{l}\text { Retrospective, } \\
\text { single-centre } \\
\text { cohort. } \\
\text { Large number of } \\
\text { exclusion criteria. } \\
\text { Outcomes limited to } \\
\text { respiratory deaths. }\end{array}$ \\
\hline
\end{tabular}




\begin{tabular}{|c|c|c|c|c|c|c|c|c|}
\hline $\begin{array}{l}\text { First author, } \\
\text { date [ref], } \\
\text { origin }\end{array}$ & Description & $\begin{array}{l}\text { Study population and } \\
\text { attrition }\end{array}$ & $\begin{array}{l}\text { CPET method and } \\
\text { CPET parameters }\end{array}$ & Exclusion & Disease outcomes & $\begin{array}{l}\text { Statistical methods to } \\
\text { investigate CPET and } \\
\text { outcome }\end{array}$ & $\begin{array}{l}\text { Summary of key reported } \\
\text { outcomes }\end{array}$ & Comments \\
\hline $\begin{array}{l}\text { FELL } 2009 \text { [24], } \\
\text { USA }\end{array}$ & $\begin{array}{l}\text { Retrospective study } \\
\text { evaluating prognostic } \\
\text { value of CPET in IPF. } \\
\text { Mean follow-up not } \\
\text { reported. }\end{array}$ & $\begin{array}{l}117 \text { IPF pts. } 10 \mathrm{pts} \\
\text { excluded from } \\
\text { survival analysis as } \\
\mathrm{VO}_{2} \text { max changed } \\
\text { between baseline } \\
\text { and } 6 \text { months. }\end{array}$ & $\begin{array}{l}\text { Cycle ergometer. } \\
\text { Blood gas } \\
\text { analysis. Peak } \\
\mathrm{VO}_{2} \cdot \mathrm{kg}^{-1}\end{array}$ & $\begin{array}{l}\text { Patients with CTD, } \\
\text { occupational or } \\
\text { environmental exposure, } \\
\text { histological pattern other } \\
\text { than UIP. }\end{array}$ & Survival & $\begin{array}{l}\text { Multivariate Cox proportional } \\
\text { hazard models studied the } \\
\text { predictive value of peak } \\
\mathrm{VO}_{2} \text { adjusting for age, } \\
\text { gender, smoking status, } \\
\text { baseline FVC\% and } \\
\text { baseline } \mathrm{DLC} \% \text {. Resulting } \\
\mathrm{HR} \text { were plotted against } \\
\text { peak } \mathrm{VO}_{2} \text { to determine } \\
\text { thresholds. Survival } \\
\text { thresholds examined with } \\
\text { Kaplan-Meier survival } \\
\text { curves, log-rank tests and } \\
\text { multivariate Cox } \\
\text { proportional hazard } \\
\text { models. }\end{array}$ & $\begin{array}{l}\text { Peak } \mathrm{VO}_{2} \cdot \mathrm{kg}^{-1} \text { examined as a } \\
\text { continuous variable did not } \\
\text { predict survival HR } 0.969 \\
(p=0.55) \text {. } \\
\text { Baseline threshold peak } \mathrm{VO}_{2} \\
<8.3 \mathrm{~mL} \cdot \mathrm{kg}^{-1} \cdot \mathrm{min}^{-1} \text { was } \\
\text { associated with an increased } \\
\text { risk of death (n=8; } \mathrm{HR} 3.24 \text {, } \\
1.10-9.56 \mathrm{Cl}, \mathrm{p}=0.03) \text {. } \\
\text { No other } \mathrm{CPET} \text { variables reported. }\end{array}$ & $\begin{array}{l}\text { Retrospective, } \\
\text { single-centre } \\
\text { study. } \\
\text { Number of deaths in } \\
\text { each group not } \\
\text { reported. } \\
\text { Analysis was not by a } \\
\text { priori plan. Small } \\
\text { number of pts } \\
\text { below } \mathrm{VO}_{2} \text { max } \\
\text { threshold in } \\
\text { analysis. } \\
\text { Caution in interpreting } \\
\text { generalisability to } \\
\text { IPF population as } \\
64 \% \text { (75/117) } \\
\text { required a surgical } \\
\text { lung biopsy for } \\
\text { diagnosis. No other } \\
\text { CPET outcomes } \\
\text { reported. }\end{array}$ \\
\hline $\begin{array}{l}\text { WaLLAERT } 2011 \\
\text { [22], France }\end{array}$ & $\begin{array}{l}\text { Retrospective } \\
\text { multicentre study } \\
\text { evaluating prognostic } \\
\text { role of CPET in } \\
\text { determining 3-year } \\
\text { survival in IPF. }\end{array}$ & 63 IPF patients & $\begin{array}{l}\text { Cycle ergometer. } \\
\text { Blood gas } \\
\text { analysis. Peak } \\
\mathrm{VO}_{2} \cdot \mathrm{kg}^{-1}, \mathrm{VE} / \mathrm{VO}_{2} \\
\text { at ventilatory } \\
\text { threshold, } \mathrm{VE} / \mathrm{CO}_{2} \text {, } \\
\text { (VOO } / \mathrm{HRR}) \mathrm{P}(\mathrm{A}-\mathrm{a}) \\
\mathrm{O}_{2} \text {, ventiliatory } \\
\text { reserve and } \\
\text { lactate. }\end{array}$ & $\begin{array}{l}\text { Non-IPF associated ILD. Pts } \\
\text { in which blood gas } \\
\text { analysis had not been } \\
\text { performed. }\end{array}$ & $\begin{array}{l}\text { 3-year survival labsence } \\
\text { of } D \text { or } L T x \text {. }\end{array}$ & $\begin{array}{l}\text { Demographic data, resting } \\
\text { pulmonary function and } \\
\text { CPET parameters in the } \\
\text { survivors were compared } \\
\text { to those who died/received } \\
\text { lung transplantation by } \\
\text { univariate survival analysis. } \\
\text { Multivariate logistic } \\
\text { regression analysis } \\
\text { explored prognosis at } \\
3 \text { years. Kaplan-Meier } \\
\text { curve and log-rank test } \\
\text { was performed, with } \\
\text { model validation by ROC } \\
\text { curve analysis. }\end{array}$ & 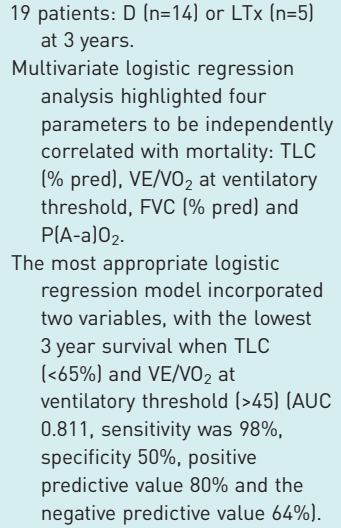 & $\begin{array}{l}\text { Retrospective study. } \\
\text { Presence of PH not } \\
\text { studied. } \\
\text { Inadequate description } \\
\text { of exclusion } \\
\text { criteria. }\end{array}$ \\
\hline
\end{tabular}




\begin{tabular}{|c|c|c|c|c|c|c|c|c|}
\hline $\begin{array}{l}\text { First author, } \\
\text { date [ref], } \\
\text { origin }\end{array}$ & Description & $\begin{array}{l}\text { Study population and } \\
\text { attrition }\end{array}$ & $\begin{array}{l}\text { CPET method and } \\
\text { CPET parameters }\end{array}$ & Exclusion & Disease outcomes & $\begin{array}{l}\text { Statistical methods to } \\
\text { investigate CPET and } \\
\text { outcome }\end{array}$ & $\begin{array}{l}\text { Summary of key reported } \\
\text { outcomes }\end{array}$ & Comments \\
\hline $\begin{array}{l}\text { GLÄSER } 2013 \\
\text { [18], } \\
\text { Germany }\end{array}$ & $\begin{array}{l}\text { Retrospective study } \\
\text { evaluating predictive } \\
\text { value of CPET } \\
\text { measures for the } \\
\text { presence of PH in } \\
\text { IPF. Follow-up } \\
2 \text { years. }\end{array}$ & $\begin{array}{l}135 \text { pts (73 with PH) } \\
\text { IPF. } \\
\text { No follow-up data } \\
\text { for } 2 \text { pts, reducing } \\
\text { cohort to } 133 \text {. }\end{array}$ & $\begin{array}{l}\text { Cycle ergometer, } \\
\text { pulse oximetry. } \\
\text { Peak } \mathrm{VO}_{2}, \mathrm{VO}_{2} \text { at } \\
\mathrm{AT}\left(\mathrm{mL} \cdot \mathrm{min}^{-1}\right) \text {, } \\
\mathrm{VE} / \mathrm{MVV}, \mathrm{VE} \text { versus } \\
\mathrm{VCO}_{2} \text { slope, VE } \\
\text { max, Vt max, Vt } \\
\text { max/IC, VE/MVV. }\end{array}$ & $\begin{array}{l}\text { Pts with left heart disease } \\
\text { (ECHO } \pm \text { PWP }>14 \mathrm{mmHg} \\
\text { by RHC), non-IPF } \\
\text { pulmonary fibrosis and/or } \\
\text { PH resulting in a life } \\
\text { expectancy } 224 \text { months, } \\
\text { inability to perform CPET } \\
\text { due to orthopaedic or } \\
\text { neurological impairment. }\end{array}$ & $\begin{array}{l}\text { Interceding pulmonary } \\
\text { hypertension. Survival } \\
\text { (death and lung } \\
\text { transplantation } \\
\text { combined endpoint) }\end{array}$ & $\begin{array}{l}\text { Mann-Whitney or chi-squared } \\
\text { test used for comparison } \\
\text { of IPF pts with/without PH. } \\
\text { Cox proportional hazards } \\
\text { analysis used for } \\
\text { pulmonary variables and } \\
\text { endpoint. Kaplan-Meier } \\
\text { survival plots constructed } \\
\text { with differences in survival } \\
\text { analysed by log-rank test. } \\
\text { Cut-off values for best } \\
\text { discrimination determined } \\
\text { using ROC curve analysis. }\end{array}$ & 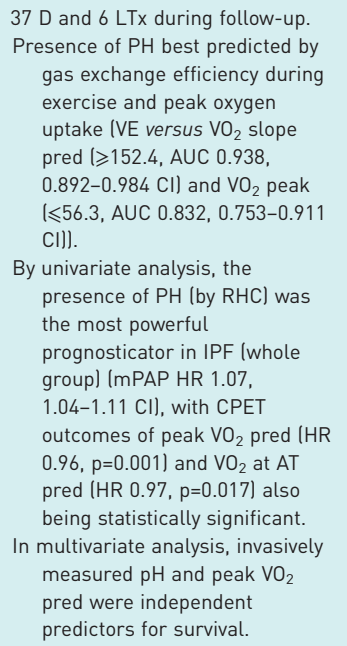 & $\begin{array}{l}\text { Retrospective } \\
\text { multicentre study. } \\
\text { Potential recruitment } \\
\text { bias due to } \\
\text { selected cohort } \\
\text { (specialist centres, } \\
\text { excluded left heart } \\
\text { diseasel. }\end{array}$ \\
\hline $\begin{array}{l}\text { VAN DER PLAS } \\
2014[20], \\
\text { Netherlands }\end{array}$ & $\begin{array}{l}\text { Retrospective study } \\
\text { exploring predictive } \\
\text { value of CPET and } \\
\text { ECHO parameters } \\
\text { for survival in IPF. } \\
\text { Mean follow-up } \\
42.3 \pm 42.2 \text { months. }\end{array}$ & $\begin{array}{l}38 \text { pts with IPF. } \\
\text { Follow-up for } 3 \text { pts } \\
\text { who received } \\
\text { transplantation } \\
\text { was censored at } \\
\text { date of } \\
\text { transplantation. }\end{array}$ & 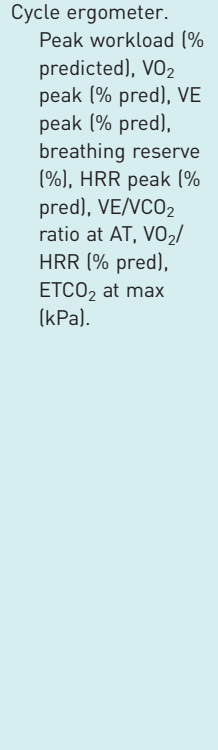 & $\begin{array}{l}\text { Non-IPF ILD. Pts where } \\
\text { CPET and ECHO were } \\
\text { performed more than } \\
2 \text { weeks apart. }\end{array}$ & Survival & $\begin{array}{l}\text { Pearson's correlation } \\
\text { coefficients were } \\
\text { calculated for sPAP and } \\
\text { CPET parameters. Patients } \\
\text { were grouped into those } \\
\text { with/without sPAP } \\
\geqslant 40 \mathrm{mmHH} \text { and differences } \\
\text { in exercise parameters } \\
\text { analysed with unpaired } \\
t \text {-test or chi-squared test. } \\
\text { ROC curve analysis was } \\
\text { used to determine } \\
\text { variables that predict sPAP } \\
\geqslant 40 \mathrm{mmHH} \text {. Kaplan-Meier } \\
\text { survival curves then } \\
\text { evaluated the prognostic } \\
\text { value of these parameters } \\
\text { on survival. HRs were } \\
\text { calculated using } \\
\text { multivariate Cox } \\
\text { proportional hazard } \\
\text { models (with FVC and CPI } \\
\text { included in the model to } \\
\text { correct for functional } \\
\text { severity of IPF) to } \\
\text { determine predictive value } \\
\text { of parameters on survival. }\end{array}$ &  & $\begin{array}{l}\text { Retrospective analysis } \\
\text { of prospective } \\
\text { database. } \\
\text { Single centre. }\end{array}$ \\
\hline
\end{tabular}




\begin{tabular}{|c|c|c|c|c|c|c|c|c|}
\hline $\begin{array}{l}\text { First author, } \\
\text { date [ref], } \\
\text { origin }\end{array}$ & Description & $\begin{array}{l}\text { Study population and } \\
\text { attrition }\end{array}$ & $\begin{array}{l}\text { CPET method and } \\
\text { CPET parameters }\end{array}$ & Exclusion & Disease outcomes & $\begin{array}{l}\text { Statistical methods to } \\
\text { investigate CPET and } \\
\text { outcome }\end{array}$ & $\begin{array}{l}\text { Summary of key reported } \\
\text { outcomes }\end{array}$ & Comments \\
\hline $\begin{array}{l}\text { KoLLERT } 2011 \\
\text { [25], } \\
\text { Germany }\end{array}$ & $\begin{array}{l}\text { Retrospective study } \\
\text { evaluating whether } \\
\text { gas exchange during } \\
\text { CPET reflects } \\
\text { disease activity and } \\
\text { clinical course in } \\
\text { sarcoidosis. } 2 \text { year } \\
\text { follow-up. }\end{array}$ & $\begin{array}{l}149 \text { histologically } \\
\text { confirmed } \\
\text { sarcoidosis. } \\
\text { Analysis of } 102 \\
\text { patients ( } 47 \\
\text { incomplete notes). }\end{array}$ & $\begin{array}{l}\text { Cycle ergometer, } \\
\text { capillary blood } \\
\text { gas analysis. } \\
\mathrm{P}(\mathrm{A}-\mathrm{a}) \mathrm{O}_{2}\end{array}$ & $\begin{array}{l}\text { Patients who could not } \\
\text { complete CPET >6 min, in } \\
\text { the absence of } \\
\text { extra-cardiopulmonary } \\
\text { limitations. Patients with } \\
\text { clinical signs of acute } \\
\text { infection. For the } \\
\text { longitudinal subgroup } \\
\text { analysis: patients with } \\
\text { incomplete records. }\end{array}$ & $\begin{array}{l}\text { Longitudinal component: } \\
\text { duration of } \\
\text { immunosuppressive } \\
\text { therapy (no treatment, } \\
\text { treatment } \leqslant 1 \text { year, } \\
\text { treatment }>1 \text { year) }\end{array}$ & $\begin{array}{l}\text { Associations between } \\
\text { sarcoidosis clinical } \\
\text { parameters lincluding the } \\
\text { need for prolonged } \\
\text { immunosuppressive } \\
\text { therapy >1 yearl and } \\
\mathrm{P}(\mathrm{A}-\mathrm{a}) \mathrm{O}_{2} \text { during exercise } \\
\text { were assessed by analysis } \\
\text { of variance statistical } \\
\text { methodology. } \\
\text { Univariate then multivariate } \\
\text { backward binary logistic } \\
\text { regression analysis used to } \\
\text { assess clinical variables } \\
\text { independently associated } \\
\text { with need for prolonged } \\
\text { immunosuppression. }\end{array}$ & $\begin{array}{l}\text { Multivariate regression analysis } \\
\text { suggested FVC (OR } 0.954 \text {, } \\
0.917-0.992 \mathrm{Cl}, \mathrm{p}=0.009) \text { and } \\
\mathrm{P}(\mathrm{A}-\mathrm{a}) \mathrm{O}_{2}(\mathrm{OR} 1.098,1.039-1.160 \\
\mathrm{Cl}, \mathrm{p}<0.0001) \text { during exercise } \\
\text { were independently associated } \\
\text { with a need for prolonged } \\
\text { immunosuppressive treatment. } \\
\text { No other } \mathrm{CPET} \text { variables reported. }\end{array}$ & $\begin{array}{l}\text { No other CPET } \\
\text { variables described } \\
\text { in analysis and } \\
\text { thus potential for } \\
\text { reporting bias. } \\
\text { Unable to determine } \\
\text { exact clinical } \\
\text { characteristics of } \\
\text { this longitudinal } \\
\text { cohort from the } \\
\text { data presented. }\end{array}$ \\
\hline $\begin{array}{l}\text { LoPEs } 2012 \\
\text { [26], Brazil }\end{array}$ & $\begin{array}{l}\text { Retrospective study to } \\
\text { identify CPET } \\
\text { measures that } \\
\text { predict FVC and } \\
\text { DLCO progression } \\
\text { over } 5 \text { years in } \\
\text { patients with thoracic } \\
\text { sarcoidosis. }\end{array}$ & $\begin{array}{l}42 \text { pts with } \\
\text { histologically } \\
\text { confirmed } \\
\text { sarcoidosis. }\end{array}$ & $\begin{array}{l}\text { Cycle ergometer, } \\
\text { blood gas } \\
\text { analysis. Peak } \mathrm{VO}_{2} \\
\text { (\% pred), \% peak } \\
\mathrm{VO}_{2} \text { at lactate } \\
\text { threshold, } \mathrm{VCO}_{2} / \\
\mathrm{VO}_{2}, \mathrm{VO}_{2} / \mathrm{HRR} \\
\text { maximum } \\
\text { respiratory rate, } \\
\text { breathing reserve, } \\
\mathrm{HRR}, \mathrm{P}(\mathrm{A} \text {-a) } \\
\mathrm{\Delta S} \mathrm{O}_{2}, \\
\Delta \mathrm{SO}_{2}, \Delta \text { lactate. }\end{array}$ & $\begin{array}{l}\text { History of smoking. } \\
\text { Mycobacterial infection, } \\
\text { exposure to } \\
\text { aero-contaminants or } \\
\text { medications known to } \\
\text { cause granulomatous } \\
\text { disorders. Those with } \\
\text { known medical history or } \\
\text { laboratory diagnosis of } \\
\text { concomitant respiratory, } \\
\text { cardiac or neuromuscular } \\
\text { disease. }\end{array}$ & $\begin{array}{l}\text { Decline FVC\% and } \\
\text { DLCO\% }\end{array}$ & $\begin{array}{l}\text { FVC/DLCO variation over study } \\
\text { period evaluated by } \\
\text { Wilcoxon signed rank test. } \\
\text { Correlations between CPET } \\
\text { measures and FVC/DLCO } \\
\text { variation over } 5 \text { years used } \\
\text { Spearman's rank } \\
\text { correlation lexcept } \\
\text { breathing reserve and } \\
\text { relative variations of FVCI. } \\
\text { ROC curve analysis used to } \\
\text { determine cut-offs for } \\
\text { CPET measurements are } \\
\text { predictors for lung } \\
\text { function decline. MLR used } \\
\text { to identify factors } \\
\text { independently related to } \\
\text { decreased lung function. }\end{array}$ & 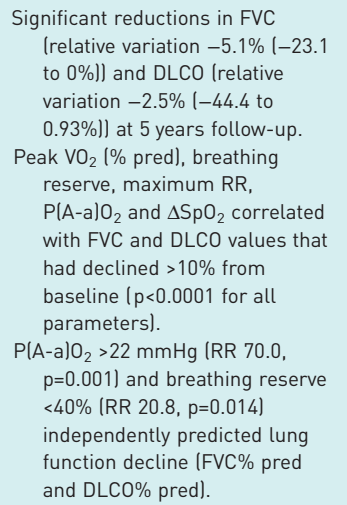 & $\begin{array}{l}\text { Retrospective, } \\
\text { single-centre study. } \\
\text { Potential for selection } \\
\text { bias (tertiary centre } \\
\text { for sarcoid - more } \\
\text { likely to have } \\
\text { severe patients). } \\
\text { Small number of } \\
\text { patients resulting in } \\
\text { high RR values. } \\
\text { Cardiac circulatory } \\
\text { status not } \\
\text { determined. }\end{array}$ \\
\hline
\end{tabular}




\begin{tabular}{|c|c|c|c|c|c|c|c|c|}
\hline $\begin{array}{l}\text { First author, } \\
\text { date [ref], } \\
\text { origin }\end{array}$ & Description & $\begin{array}{l}\text { Study population and } \\
\text { attrition }\end{array}$ & $\begin{array}{l}\text { CPET method and } \\
\text { CPET parameters }\end{array}$ & Exclusion & Disease outcomes & $\begin{array}{l}\text { Statistical methods to } \\
\text { investigate CPET and } \\
\text { outcome }\end{array}$ & $\begin{array}{l}\text { Summary of key reported } \\
\text { outcomes }\end{array}$ & Comments \\
\hline $\begin{array}{r}\text { Larton } 2017 \\
\text { [7], USA }\end{array}$ & $\begin{array}{l}\text { Retrospective study } \\
\text { evaluating predictive } \\
\text { value of CPET for } \\
\text { 1-year transplant- } \\
\text { free survival in a } \\
\text { population of ILD } \\
\text { patients undergoing } \\
\text { lung transplant } \\
\text { evaluation. }\end{array}$ & $\begin{array}{l}192 \text { pts had CPET } \\
\text { performed on } \\
\text { oxygen. Four tests } \\
\text { terminated due to } \\
\text { oxygen } \\
\text { desaturation (nadir } \\
\mathrm{SpO}_{2}<80 \% \text { despite } \\
30 \% \mathrm{FiO}_{2} \text { l. Three } \\
\text { tests terminated } \\
\text { early due to low } \\
\mathrm{ETCO}_{2}(<18 \mathrm{mmHg} \text { ) } \\
\text { or elevated } \mathrm{ETCO}{ }_{2} \\
\text { (>60 } \mathrm{mmHH} \text { ). } \\
\text { reducing cohort to } \\
185 \mathrm{pts} \text {. }\end{array}$ & $\begin{array}{l}\text { Cycle ergometer, } \\
\text { pulse oximetry. } \\
\text { Peak VO } \mathrm{O}_{2} \\
\left(\mathrm{~mL} \cdot \mathrm{kg}^{-1} \cdot \mathrm{min}^{-1} \text {, }\right. \\
\% \text { predicted), } \\
\text { workload (watts, } \\
\% \text { predicted), } \\
\mathrm{VE} / \mathrm{NCO}_{2} \text { slope } \\
\text { (\% predicted), } \\
\mathrm{ETCO}_{2} \mathrm{mmHg} \\
\text { and } \mathrm{O}_{2} \text { pulse. }\end{array}$ & $\begin{array}{l}\text { Pts not being evaluated for } \\
\text { lung transplant, those that } \\
\text { did not require oxygen } \\
\text { with exercise, no follow-up } \\
\text { data available at } 1 \text { year } \\
\text { post-CPET. }\end{array}$ & $\begin{array}{l}\text { Survival without the need } \\
\text { for transplantation lat } \\
1 \text { yearl. }\end{array}$ & $\begin{array}{l}\text { Comparison of variables } \\
\text { between those who died / } \\
\text { transplanted (D/LTx) and } \\
\text { those who survived } \\
\text { transplant-free were } \\
\text { compared using } \\
\text { two-sample independent } \\
\text { t-test. Survival was } \\
\text { calculated by } \\
\text { Kaplan-Meier method, } \\
\text { with univariable Cox } \\
\text { regression analysis to } \\
\text { identify predictors of } 1 \text { year } \\
\text { transplant-free survival. } \\
\text { Multivariable Cox model } \\
\text { with forward stepwise } \\
\text { elimination method to } \\
\text { identify prediction of } \\
\text { transplant-free survival } \\
\text { land to predict survival } \\
\text { excluding those } \\
\text { transplanted. ROC used to } \\
\text { test thresholds of these } \\
\text { predictors. }\end{array}$ &  & $\begin{array}{l}\text { Retrospective, } \\
\text { single-centre } \\
\text { cohort. } \\
\text { Potential for selection } \\
\text { bias, unidentified } \\
\text { confounding and } \\
\text { missing covariate } \\
\text { data. } \\
\text { Generalisability to } \\
\text { general ILD } \\
\text { patients } \\
\text { questionable as } \\
\text { highly selected } \\
\text { cohort of advanced } \\
\text { ILD patients. } \\
\text { Source population, } \\
\text { patterns of referral } \\
\text { transplant, waiting } \\
\text { times and cohort } \\
\text { characteristics may } \\
\text { differ from other } \\
\text { transplant } \\
\text { programmes. }\end{array}$ \\
\hline $\begin{array}{l}\text { KAWUT } 2005 \\
\text { [19], USA }\end{array}$ & $\begin{array}{l}\text { Retrospective study of } \\
\text { CPET and 6MWTD } \\
\text { variables associated } \\
\text { with survival in pts } \\
\text { referred for lung } \\
\text { transplant. Median } \\
\text { follow-up 271 days } \\
\text { (23-983). }\end{array}$ & $\begin{array}{l}51 \text { pts with IIP or } \\
\text { DPLD of known } \\
\text { cause le.g. drugs, } \\
\text { occupational or } \\
\text { environmental } \\
\text { exposures, CTD) } \\
\text { referred for lung } \\
\text { transplant. }\end{array}$ & $\begin{array}{l}\text { Cycle ergometer. } \\
\text { Pulse oximetry. } \\
\mathrm{SaO}_{2} \text { (unloaded, } \\
\text { peak, recovery), } \\
\text { peak } \mathrm{VO}_{2} \cdot \mathrm{kg}^{-1} \text {, } \\
\mathrm{VO}_{2} / \mathrm{HR}^{2} \text { peak, } \\
\mathrm{VCO}_{2} \text { unloaded, } \\
\mathrm{VE} \text { unloaded. }\end{array}$ & $\begin{array}{l}\text { Pts evaluated at another lung } \\
\text { transplantation centre. } \\
\text { Other forms of DPLD, e.g. } \\
\text { LAM, pulmonary } \\
\text { Langerhans cell } \\
\text { histiocytosis/histiocytosis } \\
\text { X, EP and granulomatous } \\
\text { DPLD, e.g. sarcoidosis. }\end{array}$ & $\begin{array}{l}\text { All-cause mortality. } \\
\text { Death on the lung } \\
\text { transplantation } \\
\text { waiting list. }\end{array}$ & $\begin{array}{l}\text { Cox proportional hazards } \\
\text { regression to identify } \\
\text { predictors of } \\
\text { time-to-death. Individual } \\
\text { models were constructed } \\
\text { using LTx as a } \\
\text { time-dependent covariate } \\
\text { to "control" for receiving a } \\
\text { LTx. ROC curve analysis } \\
\text { was used to define cut-off } \\
\text { for variables associated } \\
\text { with dying on the } \\
\text { transplantation list. }\end{array}$ & 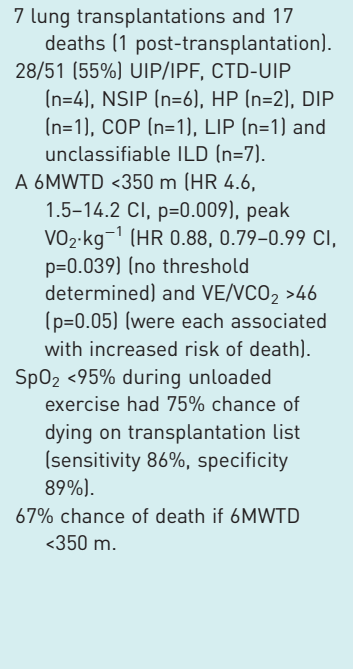 & $\begin{array}{l}\text { Retrospective } \\
\text { single-centre } \\
\text { cohort.Onty half pts } \\
\text { reached AT which } \\
\text { limited analysis } \\
\text { (low number of } \\
\text { endpoints). } \\
\text { Additional oxygen use } \\
\text { during CPET was } \\
\text { variable. } \\
\text { Generalisability } \\
\text { questionable as } \\
\text { highly selected } \\
\text { cohort of severe } \\
\text { ILD. } \\
\text { Source population, } \\
\text { patterns of referral } \\
\text { to transplant } \\
\text { centre, witing } \\
\text { times and cohort } \\
\text { characteristics may } \\
\text { differ from other } \\
\text { transplant } \\
\text { programmes. }\end{array}$ \\
\hline
\end{tabular}




\begin{tabular}{|c|c|c|c|c|c|c|c|c|}
\hline $\begin{array}{l}\text { First author, } \\
\text { date [ref], } \\
\text { origin }\end{array}$ & Description & $\begin{array}{l}\text { Study population and } \\
\text { attrition }\end{array}$ & $\begin{array}{l}\text { CPET method and } \\
\text { CPET parameters }\end{array}$ & Exclusion & Disease outcomes & $\begin{array}{l}\text { Statistical methods to } \\
\text { investigate CPET and } \\
\text { outcome }\end{array}$ & $\begin{array}{l}\text { Summary of key reported } \\
\text { outcomes }\end{array}$ & Comments \\
\hline $\begin{array}{r}\text { SwIGRIS } 2009 \\
\text { [27], USA }\end{array}$ & $\begin{array}{l}\text { Retrospective study } \\
\text { exploring prognostic } \\
\text { role of } \mathrm{SpO}_{2} \text { and } \\
\mathrm{SaO}_{2} \text { at rest and } \\
\text { during maximal } \\
\text { exercise in } \mathrm{SSc}-\mathrm{ILD} \\
\text { exercise. Median } \\
\text { follow-up } 7.1 \text { years. }\end{array}$ & $\begin{array}{l}83 \text { patients with } \\
\text { SSc-ILD }\end{array}$ & $\begin{array}{l}\text { Cycle ergometer. } \\
\text { Blood gas } \\
\text { analysis and pulse } \\
\text { oximetry. } \mathrm{SpO}_{2} \\
\text { and } \mathrm{SaO}_{2} \text { at rest } \\
\text { and during } \\
\text { maximal exercise } \\
\mathrm{SSpO}_{2} \text { max). } \mathrm{V}_{2} \\
\text { max measured } \\
\text { but not reported. }\end{array}$ & $\begin{array}{l}\text { Pulmonary hypertension, } \\
\text { overlap syndromes. }\end{array}$ & Mortality & $\begin{array}{l}\text { Cox proportional hazard } \\
\text { models were used to } \\
\text { examine the prognostic } \\
\text { capabilities of } \mathrm{SpO}_{2} \text {, } \\
\text { dichotomised by }<89 \% \text { or } \\
\geqslant 89 \% \text { and also as } \\
\text { continuous variables. } \\
\text { Kaplan-Meier survival } \\
\text { curves were generated. }\end{array}$ & 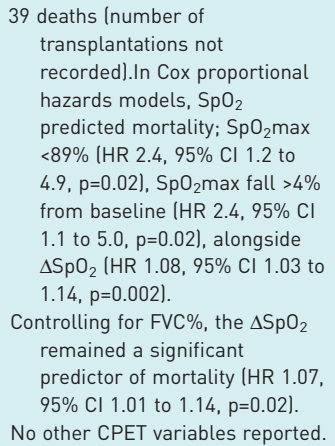 & $\begin{array}{l}\text { No other CPET } \\
\text { variables described } \\
\text { in analysis and } \\
\text { thus potential for } \\
\text { reporting bias. }\end{array}$ \\
\hline
\end{tabular}

Abbreviations: $\triangle \mathrm{SpO}_{2}$ : difference between peak and resting oxygen saturation; 6MWTD: 6-minute walk test distance; AaDO $_{2}$ : alveolar-arterial oxygen pressure difference; AT: anaerobic threshold; AUC: area under the curve; BR: breathing reserve [1 - (VE during exercise/MVV)] × 100; Cl: confidence interval; COP: cryptogenic organising pneumonia; CPET: cardiopulmonary exercise testing; CPI: composite physiologic index; CTD: connective tissue disease; CXR: chest X-ray; D: died/deaths; DLCO: diffusion capacity of lungs for carbon dioxide; DPLD: diffuse parenchymal lung disease; ECHO: echocardiogram; EP: eosinophilic pneumonia; $\mathrm{ETCO}_{2}$ : end tidal carbon dioxide; FiO ${ }_{2}$ : fraction of inspired oxygen; FVC: forced vital capacity; HP: hypersensitivity pneumonitis; HR: hazard ratio; HRCT: high-resolution computed tomography; HRR: heart rate; IC: inspiratory capacity; ILD: interstitial lung disease; IPF: idiopathic pulmonary fibrosis; LAM: lymphangioleiomyomatosis; LTx: lung transplantation; max: maximal; MLR: multiple logistic regression; MVV: maximum voluntary ventilation (can be measured or estimated as FEV1 × 41); OR: odds ratio; $\mathrm{PaCO}_{2}$ : partial pressure of carbon dioxide; $\mathrm{PaO}_{2}$ : partial pressure of oxygen; pts: patients; NSIP: non-specific interstitial pneumonia; $\mathrm{P}\left(\mathrm{A}-\mathrm{a} \mathrm{O}_{2}\right.$ : alveolar-arterial oxygen pressure gradient at peak exercise; PH: pulmonary hypertension; pred: predicted; PVD: peripheral vascular disease; PWP: pulmonary capillary wedge pressure; RCT: randomised controlled trial; $\mathrm{RHC}$; right heart catheter; $\mathrm{ROC}$ : receiver operating characteristic curve; $\mathrm{RR}$ : respiratory rate; SaO $\mathrm{O}_{2}$ oxygen saturation of arterial blood; SPAP: systolic pulmonary artery pressure; $\mathrm{SpO}_{2}$ : oxygen saturation measured by pulse oximetry; SSc: systemic sclerosis; TLC: total lung capacity; UIP: usual interstitial pneumonia; $\mathrm{VCO}_{2}$ : carbon dioxide production; $\mathrm{VD} / \mathrm{VT}$ : physiological dead space/tidal volume ratio; $\mathrm{VE}$ : minute ventilation; $\mathrm{VE} / \mathrm{VCO} \mathrm{O}_{2}$ : ventilatory equivalent for carbon dioxide; $\mathrm{VE} / \mathrm{VO}_{2}$ : ventilatory equivalent for oxygen; $\mathrm{VO}_{2}$ : oxygen uptake; $\mathrm{VO}_{2}$ slope: $\mathrm{PaO}_{2}$ plotted against $\mathrm{VO}_{2} ; \mathrm{VO}_{2} / \mathrm{HRR}$ max or oxygen pulse: oxygen delivery per heartbeat; $\mathrm{VT}$ : ventilatory threshold (highest $\mathrm{VO}_{2}$ sustained without lactic acidosis); $\mathrm{Vt}$ : tidal volume; tidal volume reserve: $\mathrm{Vt}$ max-Vt resting. 


\section{Patient populations}

The majority $(8 / 13,62 \%)$ exclusively recruited patients with IPF, with retrospective assessment of 703 IPF patients and prospective assessment of 59 IPF patients. Classification of IPF was based on accepted criteria used at the time of enrolment: the 2000 American Thoracic Society (ATS) international consensus (IC) statement $[1,20,21,23,24,28]$ and the later 2002 ATS/ERS (European Respiratory Society) IC classification of the idiopathic interstitial pneumonias (including IPF) $[19,22,30]$. The updated 2011 ATS/ERS/JRS/ALAT guidelines for the diagnosis of IPF [31] were applied in all [7, 18, 28, 29] but one of the studies [20] published after 2011 (the latter was a retrospective study that may have recruited patients prior to the 2011 guidelines).

Two retrospective studies explored the prognostic role of CPET in 144 histologically confirmed sarcoidosis patients [25, 26], representing Scadding disease stages 1-4 [32]. Only one retrospective study had examined the prognostic role of CPET in systemic sclerosis ILD (SSc-ILD) ( $n=83$ ) [27]. Patients with SSc met classification criteria adopted by the 1980 American Rheumatology Association [33] and those with SSc sine scleroderma met criteria proposed by Poormoghim and colleagues [34]. A diagnosis of ILD was based on chest radiography in 60/83 patients [27].

The prognostic role of CPET in other secondary causes of ILD (such as myositis, occupational causes of ILD and hypersensitivity pneumonitis (HP)) and/or other forms of idiopathic interstitial pneumonias (IIP) has not been well studied. Two retrospective studies have reported the prognostic value of CPET in mixed ILD populations referred for lung transplantation [7, 19], but low patient numbers precluded useful subgroup analyses.

The majority of studies had a moderate $(6 / 13,46 \%)$ or high $(4 / 13,31 \%)[7,19,21,25]$ risk of bias for participant selection. For example, generalisability in one study was limited by the lack of clearly defined clinical characteristics (e.g. Scadding disease stage) in patients followed longitudinally (102/149) [30]. Studies enrolling from populations referred for lung transplantation resulted in selected cohorts of advanced ILD patients [7, 19]. Others incorporated a priori patient grouping, for example the presence of pulmonary hypertension $(\mathrm{PH})$ [18], to enrich populations with those at higher risk of outcomes of interest, or actively excluded relevant patients, e.g. those that died from a cause other than respiratory failure [21].

Study attrition was generally low, consistent with the retrospective nature of the majority of studies. The QUIPS risk of bias for study attrition was high in two studies. Over $25 \%$ of patients were excluded from the analyses by Lopes et al. [26] (due to smoking history, concomitant respiratory disease, cardiac disease and neuromuscular disease). In another study, 34\% (80/238) of the original study population were excluded from the analysis because of incomplete data sets [23].

\section{Prognostic factor measurement}

CPET was the sole prognostic factor for the majority of studies $(8 / 13,62 \%)$, with a minority using CPET as part of a broader repertoire of exploratory physiological tests including 6MWT $[7,19,28]$ or lung function parameters [18]. One study incorporated CPET with other clinical, radiological and resting physiological assessments to devise a scoring system to predict survival in newly diagnosed cases of IPF (the clinical-radiological-physiological score) [23].

In two studies, CPET was the principal method of achieving maximal exercise [25, 27], with arterial blood gas sampling or peripheral oxygenation measurements used to determine the effect of exercise on gas exchange. In both studies, typical CPET measures, such as peak $\mathrm{VO}_{2}$, were not reported.

The bias rating for prognostic factor measurement using the QUIPS tool was generally low to moderate (figure 2), with the majority of studies reporting a standardised approach to CPET (albeit individualised for each study) and analysis that would be easily reproducible and not amenable to bias. Most studies provided a sufficient description of the CPET protocol used (6/10, 60\%), adhering to the 2003 ATS statement on CPET testing [7, 18-20, 22, 28]. Variance in the use of supplemental oxygen during CPET was observed; oxygen usage was an inclusion criteria in one study [7], whilst in others, supplemental oxygen was applied variably, depending on a pre-study requirement for home oxygen or saturation on room air $<90 \%$ [19]. In 7 of 13 (54\%) studies, blood gas analysis was used to assess the adequacy of gas exchange during exercise [21-27], whilst the remainder used pulse oximetry, considered by some experts to be a suboptimal substitute [13]. A broad range of quantitative CPET parameters were presented/ analysed (summarised in table 1), raising the possibility of reporting bias (see later).

All but one study used cycle ergometry. Treadmill exercise testing was used as the method of CPET in the remaining study, with exercise increments based on a patient's daily activities and parameters of resting pulmonary function; this raises concerns regarding variation amongst subjects [21]. Furthermore, inherent differences in physiological responses recorded by the two ergometers during incremental exercise have been well defined, and make direct comparison of the two methods problematic $[35,36]$. 


\begin{tabular}{|c|c|c|c|c|c|c|}
\hline First author, year & $\begin{array}{c}\text { Study } \\
\text { participation }\end{array}$ & Study attrition & $\begin{array}{l}\text { Prognostic } \\
\text { factor } \\
\text { measurement }\end{array}$ & $\begin{array}{c}\text { Outcome } \\
\text { measurement }\end{array}$ & $\begin{array}{l}\text { Study } \\
\text { confounding }\end{array}$ & $\begin{array}{l}\text { Statistical } \\
\text { analysis and } \\
\text { reporting }\end{array}$ \\
\hline \multicolumn{7}{|l|}{ KING 2001 [23] } \\
\hline \multicolumn{7}{|l|}{ MIKI 2003 [21] } \\
\hline \multicolumn{7}{|l|}{ KAWUT 2005 [19] } \\
\hline \multicolumn{7}{|l|}{$\begin{array}{l}\text { SWIGRIS } 2009 \\
\text { [27] }\end{array}$} \\
\hline \multicolumn{7}{|l|}{ FELL 2009 [24] } \\
\hline \multicolumn{7}{|l|}{$\begin{array}{l}\text { WALLAERT } 2011 \\
\text { [22] }\end{array}$} \\
\hline \multicolumn{7}{|l|}{$\begin{array}{l}\text { KOLLERT } 2011 \\
\text { [25] }\end{array}$} \\
\hline \multicolumn{7}{|l|}{ LOPES 2012 [26] } \\
\hline \multicolumn{7}{|l|}{$\begin{array}{l}\text { TRIANTAFILLIDOU } \\
2013 \text { [28] }\end{array}$} \\
\hline \multicolumn{7}{|l|}{ GLÄSER 2013 [18] } \\
\hline \multicolumn{7}{|l|}{$\begin{array}{l}\text { VAINSHELBOIM } \\
2016 \text { [29] }\end{array}$} \\
\hline $\begin{array}{l}\text { LAYTON } 2017 \\
\text { [7] }\end{array}$ & & & & & & \\
\hline
\end{tabular}

FIGURE 2 The Quality in Prognosis Study (QUIPS) risk of bias tool assessment of included studies. Green indicates low risk of bias, amber indicates a moderate risk and red indicates a high risk of bias.

\section{Outcome measurement}

Eleven of 13 studies (85\%) evaluated mortality. The majority of these (10/11,91\%) examined all-cause mortality, considering death or lung transplantation as composite endpoint. The remaining study used an outcome measurement that was restricted to respiratory deaths only [21]. One study assessed the discriminatory ability of CPET to identify patients who would die on the lung transplant list before receiving transplantation [19]. Other outcomes included interceding $\mathrm{PH}$ [18], decline in pulmonary function (FVC, forced vital capacity), decline in DLCO (diffusion capacity for carbon monoxide) and/or duration of immunosuppressive therapy in sarcoidosis $[25,26]$.

The risk of bias for outcome measure assessment was considered low to moderate across all studies (fig 2).

\section{Reported prognostic associations of CPET in ILD}

All studies reported at least one positive association between CPET and clinical outcomes, raising the possibility of positive reporting bias. A summary of the main findings is presented in table 2. Significant heterogeneity in study design, study populations (and classification criteria adopted), CPET protocols, CPET endpoints and defined endpoints precluded a meta-analysis.

\section{Maximal oxygen consumption}

The prognostic value of measures of maximal oxygen consumption during CPET on ILD outcomes have been reported in 10/13 (77\%) studies (table 2).

Peak $\mathrm{VO}_{2} \cdot \mathrm{kg}^{-1}$ inversely correlated with increased 1-year mortality in two cohorts of patients with severe ILD referred for lung transplantation [7, 19], whilst peak $\mathrm{VO}_{2}$ thresholds ranging from <8.3 to $<14.2 \mathrm{~mL} \cdot \mathrm{kg}^{-1} \cdot \mathrm{min}^{-1}[24,28,29]$ were reported to predict mortality in IPF. These results contrasted with the findings of other studies that failed to identify any significant association [20-22].

\section{Ventilatory efficiency}

The prognostic value of the ventilatory equivalent for $\mathrm{CO}_{2}$ at $\mathrm{AT}\left(\mathrm{VE} / \mathrm{VCO}_{2}\right.$ at $\mathrm{AT}$ ) at levels ranging between $>34$ and $>46$ was reported to predict survival in IPF [7, 19, 20], even after correcting for functional severity of ILD [20] (table 2).

The ventilatory equivalent for oxygen at $\mathrm{AT}\left(\mathrm{VE} / \mathrm{VO}_{2}\right.$ at $\left.\mathrm{AT}\right)$ was also reported to be a poor predictor of survival in IPF patients $[21,22]$ and whilst $\mathrm{VE} / \mathrm{VO}_{2}$ was associated with worse IPF survival in the derivation cohort of the clinical-radiological-physiological multimodal score, even after adjustment for age and smoking status, it was not included as a parameter in the final model [21]. 
TABLE 2 Reported associations between CPET parameters and outcomes in studies of ILD

\begin{tabular}{|c|c|c|c|}
\hline CPET measurement & Studies & Threshold & Outcome \\
\hline \multirow{10}{*}{$\begin{array}{l}\text { Maximal oxygen } \\
\text { consumption (peak } \\
\mathrm{VO}_{2} \text { ) }\end{array}$} & GLÄSER 2013 & Peak $\mathrm{VO}_{2 \% \text { pred }}<56.3 \%$ & Presence of $\mathrm{PH}$. \\
\hline & KawUt 2005 & $\begin{array}{l}\text { Peak VO} \mathrm{V}_{2} \cdot \mathrm{kg}^{-1} \text { (no threshold determined), } \\
\text { associated with worse outcome. }\end{array}$ & $\begin{array}{l}\text { Mortality at } 1 \text { year of mixed ILD patients } \\
\text { referred for transplantation. }\end{array}$ \\
\hline & KING 2001 & $\begin{array}{l}\text { Methodology suggested peak } \mathrm{VO}_{2} \text { was recorded but } \\
\text { result not reported in results section. }\end{array}$ & Survival in IPF. \\
\hline & KolLert 2011 & Not measured. & $\begin{array}{l}\text { Prolonged immunosuppressive therapy } \\
\text { (>1 year) in sarcoidosis. }\end{array}$ \\
\hline & LOPES 2012 & $\begin{array}{l}\text { Peak } \mathrm{VO}_{2}<50 \% \text { pred (association on univariate but } \\
\text { not multivariate analysis). }\end{array}$ & $\begin{array}{l}\text { Decline of }>10 \% \text { FVC } \% \text { pred and DLCO } \\
\% \text { pred at } 5 \text { years follow-up from } \\
\text { baseline, in thoracic sarcoidosis. }\end{array}$ \\
\hline & MІкı 2003 & $\begin{array}{l}\text { Peak } \mathrm{VO}_{2} \text { lassociated with worse outcome using } \\
\text { univariate analysis, but not on multivariate). }\end{array}$ & Respiratory deaths in IPF. \\
\hline & SWIGRIS 2009 & $\begin{array}{l}\text { Although measured, not part of planned statistical } \\
\text { analysis. }\end{array}$ & Mortality in SSc-ILD. \\
\hline & $\begin{array}{l}\text { TRIANTAFILLIDOU } \\
2013\end{array}$ & $\begin{array}{l}\text { Peak } \mathrm{VO}_{2}<14.2 \mathrm{~mL} \cdot \mathrm{kg}^{-1} \cdot \mathrm{min}^{-1} \text { associated with } \\
\text { worse outcome and further enforced when the } \\
\text { model combines } \mathrm{DLCO} \text {. }\end{array}$ & Survival in IPF. \\
\hline & VAINSHELBOIM 2016 & $\begin{array}{l}\text { Peak } \mathrm{VO}_{2}<13.8 \mathrm{~mL} \cdot \mathrm{kg}^{-1} \cdot \mathrm{min}^{-1} \text { associated with } \\
\text { worse outcome (AUC } 0.731,0.56-0.9, \mathrm{p}=0.031 \text { ). }\end{array}$ & Mortality or transplantation in IPF. \\
\hline & VAN DER PLAS 2014 & Peak $\mathrm{VO}_{2} \cdot \mathrm{kg}^{-1}-$ no association. & Survival in IPF. \\
\hline \multirow{8}{*}{$\begin{array}{l}\text { Ventilatory efficiency } \\
\left(\mathrm{VE} / \mathrm{VO}_{2}, \mathrm{VE} / \mathrm{VCO}_{2}\right)\end{array}$} & KING 2005 & $\begin{array}{l}\text { VE/VO } \\
\text { results adjusted for age and smoking status (HR } \\
\text { 1.06). Not included in multivariable model. }\end{array}$ & Survival in IPF. \\
\hline & Kollert 2011 & Not measured & $\begin{array}{l}\text { Prolonged immunosuppressive therapy } \\
\text { ( }>1 \text { year) in sarcoidosis. }\end{array}$ \\
\hline & LAYTON 2017 & $\begin{array}{l}\mathrm{VE} / \mathrm{VCO}_{2} \text { slope lassociation with univariate analysis } \\
\text { but not multivariate) }\end{array}$ & $\begin{array}{l}\text { 1-year mortality or transplantation in } \\
\text { mixed population of ILD. }\end{array}$ \\
\hline & LOPES 2012 & Not measured & $\begin{array}{l}\text { Decline in FVC and DLCO at } 5 \text { years in } \\
\text { sarcoidosis. }\end{array}$ \\
\hline & MIKI 2003 & $\begin{array}{l}\mathrm{VE} / \mathrm{VO}_{2} \text { at max } \mathrm{VE} / \mathrm{VCO}_{2} \text { at max lassociated with } \\
\text { worse outcome using univariate analysis, but not } \\
\text { on multivariate). }\end{array}$ & Respiratory deaths in IPF. \\
\hline & SWIGRIS 2009 & Not measured & Survival in SSc-ILD. \\
\hline & $\begin{array}{l}\text { TRIANTAFILLIDOU } \\
2013\end{array}$ & $\begin{array}{l}\mathrm{VE} / \mathrm{VCO}_{2} \text { slope and higher } \mathrm{VE} / \mathrm{VCO}_{2} \text { at } \mathrm{AT} \text { predicted } \\
\text { worse outcome. }\end{array}$ & Survival in IPF. \\
\hline & VAINSHELBOIM 2016 & $\begin{array}{l}\mathrm{VE} / \mathrm{VCO}_{2} \text { at } \mathrm{AT}>34 \text { and nadir } \mathrm{VE} / \mathrm{VO}_{2}>34 \text { predicted } \\
\text { worse outcome in univariate and bivariate } \\
\text { analysis }\end{array}$ & Mortality in IPF. \\
\hline
\end{tabular}




\section{CPET measurement}

Diffusion limitation or
exercise-induced
hypoxaemia

GLÄSER

KAWUT 2013

KING 2001

KOLLERT 2011

LAYTON 2017

LOPES 2012

MIKI 2003

SWIGRIS 2009

TRIANTAFILLIDOU 2013

VAINSHELBOIM 2016

VAN DER PLAS 2014

WALLAERT 2011

\section{Threshold}

$\mathrm{VE} / \mathrm{VCO}_{2}$ at $\mathrm{AT}>45$ associated with poorer survival (HR 4.58, $p=0.001$ ), even after correcting for lung function severity.

$\mathrm{VE} / \mathrm{VO}_{2}$ at $\mathrm{AT}>45$ associated with worse outcome (multivariate analysis).

Resting $\mathrm{PaO}_{2}$ was associated with worse outcome (HR 0.934) when adjusted for age, sex, baseline physiology and smoking status. No threshold could be determined.

Although $\mathrm{SpO}_{2}$ monitored during $\mathrm{CPET}$, not included in analysis.

$\mathrm{SaO}_{2}<95 \%$ during unloaded exercise lone of several variables) predicting worse outcome ( $p=0.0025$ ).

$\mathrm{SaO}_{2}<95 \%$ during unloaded exercise lone of several variables) predicting worse outcome (sens. of $86 \%$, spec. $89 \%$ ).

$\mathrm{PaO}_{2}$ at maximal exercise associated with worse outcome and included in multivariable model laccounted for as much as $10.5 \%$ of the maximum score in the model).

$\mathrm{P}(\mathrm{A}-\mathrm{a}) \mathrm{O}_{2}$ associated with worse outcome (multivariate analysis, OR 1.098, $p<0.001$ ).

Nadir CPET $\mathrm{SpO}_{2}<86 \%$ independently associated with worse outcome (HR 2.27, $p=0.001)$. Risk of death/lung transplantation increased two-fold when $\mathrm{SpO}_{2}<86 \%$.

$\mathrm{P}(\mathrm{A}-\mathrm{a}) \mathrm{O}_{2}>22 \mathrm{mmHg}$ associated with worse outcome (multivariate analysis, RR 70.0, $\mathrm{p}<0.001)$.

$\mathrm{PaO}_{2}$ slope $\left(\Delta \mathrm{PaO}_{2} / \Delta \mathrm{VO}_{2}\right)$ predicted worse outcome (multivariate analysis, HR 0.841, $\mathrm{p}=0.015$ ). Those stratified $\leqslant-60 \mathrm{mmHg} \cdot \mathrm{L}^{-1} \cdot \mathrm{min}^{-1}$ associated with worse survival ( 1.6 years versus 4.5 years).

$\mathrm{SpO}_{2}$ at maximum exercise $<89 \%$ (HR 2.4) or $\mathrm{SpO}_{2}$ at maximum exercise fall $>4$ points from baseline (HR 2.4) associated with worse outcome.

$\mathrm{SpO}_{2}$ at peak exercise - no association

Although $\mathrm{SpO}_{2}$ monitored during $\mathrm{CPET}$, not included in analysis.

Not specifically reported on.

Higher $\mathrm{P}(\mathrm{A}-\mathrm{a}) \mathrm{O}_{2}$ associated with worse outcome using multivariate analysis, but was not included in the final logistic regression model.

\section{Outcome}

Survival in IPF.

3-year survival in IPF.

Survival in IPF.

Survival in IPF or development of interceding $\mathrm{PH}$.

All-cause mortality at 1 year of mixed ILD patients referred for transplantation.

Death on waiting list for lung transplantation.

Survival in IPF.

Prolonged immunosuppressive therapy (>1 year) in sarcoidosis.

1 -year mortality or transplantation in mixed population of ILD.

Decline of $>10 \%$ FVC $\%$ pred and DLCO $\%$ pred at 5 years follow-up from baseline, in thoracic sarcoidosis.

Respiratory deaths in IPF.

Survival in SSc-ILD.

Survival in IPF.

Mortality in IPF.

Survival in IPF.

3-year survival in IPF.

Abbreviations: AT: anaerobic threshold; DLCO: diffusion capacity of lungs for carbon dioxide; FVC: forced vital capacity; HR: hazard ratio; ILD: interstitial lung disease; IPF: idiopathic pulmonary fibrosis; max: maximum; OR: odds ratio; $\mathrm{P}(\mathrm{A}-\mathrm{a}) \mathrm{O}_{2}$ : alveolar-arterial oxygen pressure gradient at peak exercise; pred: predicted; RR: relative risk; $\mathrm{SaO}_{2}$ : oxygen saturation of arterial blood; sens.: sensitivity; spec.: specificity; $\mathrm{SpO}_{2}$ : oxygen saturation measured by pulse oximetry; $\mathrm{SSc}$ : systemic sclerosis; $\mathrm{VE} / \mathrm{VCO}_{2}$ : ventilatory equivalent for carbon dioxide; $\mathrm{VE} / \mathrm{VO}_{2}$ : ventilatory equivalent for oxygen; $\mathrm{VO}_{2}$ : oxygen uptake.

\section{Diffusion limitation or exercise-induced hypoxaemia}

Exercise-induced hypoxaemia was reported as a potential prognostic factor for survival in IPF [21, 23]. $\mathrm{PaO}_{2}$ at the end of maximal exercise was the only CPET-derived parameter included in the comprehensive clinical-radiological-physiological multimodal score predicting survival in IPF, and when weighted, accounted for as much as $10.5 \%$ of the maximum score in the final model [23]. 
In mixed populations of ILD patients with advanced disease and referred for lung transplantation [7, 19], desaturation during CPET was reported to be predictive of lung transplantation or death.

In two studies examining longitudinal outcomes in sarcoidosis, the alveolar-arterial oxygen pressure gradient during exercise $\mathrm{P}(\mathrm{A}-\mathrm{a}) \mathrm{O}_{2}$ (a measure of arterial desaturation during exercise) was independently associated with both the need for prolonged ( $>1$ year) immunosuppressive therapy [25] and decline in pulmonary function at 5 years [26].

Finally, in the single study of SSc-ILD [27], akin to studies of sarcoidosis and IPF, diffusion limitation, measured in this study as the change in peripheral oxygenation $\left(\mathrm{SpO}_{2}\right)$ during CPET, correlated with survival.

\section{Study confounders}

The majority of studies were considered to be at "high" risk of bias due to inadequate account of potential confounding factors or methods of statistical analysis/reporting (fig 2). The data used in the majority of studies was obtained from existing databases and/or case note review $(85 \%, n=11)$ and as such, the contribution of potential important confounders such as comorbid disease [18, 21, 22, 24, 26, 27], body mass index [19-21, 24, 26, 28] and smoking status [18, 19, 22, 26] was not recorded. Baseline "disease severity" was only specifically addressed as a potential confounder by one study [20]. The use of variable levels of supplemental oxygen (or uncertain inspired oxygen concentrations) in some of the reviewed studies $[7,19]$ is also a major limitation that potentially impacts on the accuracy of peak $\mathrm{VO}_{2}$.

As discussed previously, studies reporting outcomes in subjects referred for transplantation reduces the generalisability of the study findings $[7,20]$, selecting cohorts of more advanced ILD patients. Other studies focused on healthier populations of ILD patients (e.g. not requiring supplemental oxygen during $\mathrm{CPET})$, and this unsurprisingly resulted in lower mortality rates $(\mathrm{n}<10)[24,28,29]$.

Multiple logistic regression (MLR) was the dominant statistical methodology used to determine the relationship between CPET parameters and clinical outcomes in ILD. Whilst this approach adjusts for the effects of known confounders, most of the study sample sizes were smaller than the proposed minimum requirement for MLR analysis [37]. Only one study reported an a priori power calculation to influence sample size [29]; others were underpowered to detect the outcomes proposed.

Stepwise multiple regression was used by some studies to determine the optimal model parameters to predict increased mortality [23, 28]. This approach uses parameter inference, which may lead to over-fitting of some parameters or exclusion of confounders that do not reach statistical significance [38]. Furthermore, the number of parameters or order entry (or deletion) can also affect the selected model [39] and affect the likelihood of type I error [38]. Only one study specifically attempted to reduce multicollinearity [23], which if overlooked can increase the risk of type II error [40].

\section{Discussion}

Clinicians would benefit from reliable prognostic markers for patients with ILD to enable timelier referral for transplantation, improved monitoring of existing therapies, and to determine the efficacy of novel treatments in clinical trials $[12,41]$.

To our knowledge, this is the first study to systematically review and critically appraise studies that have reported the prognostic value of CPET in ILD. Thirteen studies were identified; survival was the principal clinical outcome measured. The utilisation of numerous methodologies, CPET parameters and timing of mortality evaluation prevented the determination of definitive CPET thresholds for predicting outcomes in ILD. Due to the clinical diversity of the studies and moderate risk of bias in all studies in at least one domain of the QUIPS tool, meta-analysis was not possible. It was also felt that meta-analysis might overstate the findings of these small-scale, poorly matched studies.

There were conflicting results with regards to the prognostic role of maximal oxygen consumption in predicting survival, which may in part be attributable to the heterogeneity of the studies concerned. Whilst reductions in ventilatory efficiency have been reported to predict both the presence of $\mathrm{PH}$ [42] and development of interceding PH in IPF cohorts [18], an independent prognostic value in IPF patients was not determined. The magnitude of hyperventilation at ventilatory threshold does, however, warrant further exploration as a prognostic factor in ILD, particularly as a marker of concurrent cardiopulmonary vascular impairment. Exercise-induced hypoxaemia was another potential prognostic outcome reported in several studies. A study directly comparing the longitudinal prognostic value of CPET with alternative forms of exercise testing, such as 6-minute walk testing, could therefore be justified.

Issues around study design (relating primarily to the inherent problems of retrospective studies, patient selection and presentation of numerous CPET parameters), insufficient adjustment for confounding 
variables and inadequate statistical analyses limits the strength of conclusions that can be drawn from the studies undertaken to date. Whilst the associations presented shed important light on the potential role of CPET in disease prognostication in ILD, there is currently insufficient evidence to support its use in facilitating "real-world" clinical decisions and larger prospective studies are required. In planning future clinical studies, rigorously phenotyped patient cohorts, characterised using standardised definitions and with external validation or multicentre cohorts, will be imperative to try to overcome some of the challenges encountered by studying heterogeneous ILD populations.

Several practical challenges of CPET, including lack of measurement standardisation, non-uniform parameter availability from different instrument manufacturers, provision of adequate training of personnel, availability of equipment in secondary care, establishment of optimal exercise duration and ramping protocol, alongside individual patient safety considerations, such as desaturation to prohibitive levels in advanced ILD, will all need to be addressed prior to its consideration in clinical practice in ILD patient populations. The absence of sufficient longitudinal data to identify a minimally clinically important change in CPET values in ILDs is a further obstacle that will also need to be overcome $[12,43]$.

This work has identified a number of considerations for future prognostic studies of CPET in ILD. Common to many human diseases, the disease progression in ILD is probably influenced by a complex interplay of patient, genetic, environmental and treatment factors. As such, a multivariable approach to the design and analysis of future prognostic studies of ILD is essential if we are to confirm a specific role for CPET in routine monitoring. In contrast to RCTs there are no robust standards defining the need to register or publish protocols for prognostic research and as such it is not always transparent whether statistical analysis was part of the a priori plan [44]. Almost all studies in this review examined multiple prognostic CPET variables and as such there is potential for selective reporting bias that could be largely overcome by more stringent protocol registration with pre-specified outcomes of interest. It is important that relevant study confounders are taken into consideration in future studies examining the prognostic value of CPET in ILD to establish whether CPET provides additional prognostic value beyond more easily obtainable clinical and physiological outcomes.

\section{Conclusions: take-home message}

CPET may have a role as a prognostic factor in ILD but the quality of existing studies and lack of MCID values in ILDs limits the conclusions that can be drawn at present. Large carefully designed prospective studies are needed to establish the role of CPET in the longitudinal assessment of ILD in the future.

Acknowledgements: Independent statistical advice was sought from Paul White (statistician at the University of the West of England, Bristol, UK) to confirm that a meta-analysis was not possible.

Author contributions: S.L. Barratt is the guarantor of the content of the manuscript, including data and analysis. C. Sharp and R. Davis undertook the initial literature review and data extraction. This was then verified by S.L. Barrett and J.D. Pauling who prepared the manuscript. R. Davis and C. Sharp verified the manuscript.

Conflict of interest: S.L. Barratt reports personal fees for an advisory board and financial support to attend an educational conference from Boehringer Ingelheim outside the submitted work. R. Davis is an employee of Boehringer Ingelheim. Boehringer Ingelheim did not have any involvement in the research or the preparation of this manuscript. C. Sharp has nothing to disclose. J.D. Pauling reports personal fees from Boehringer Ingelheim; grants, personal fees and non-financial support for attendance at educational meetings from Actelion Pharmaceuticals; and personal fees from Sojournix Pharma, all outside the submitted work.

\section{References}

1 American Thoracic Society. Idiopathic Pulmonary Fibrosis: Diagnosis and Treatment. International Consensus Statement. American Thoracic Society (ATS), and the European Respiratory Society (ERS). Am J Respir Crit Care Med 2000; 161: 646-664.

2 Bellaye PS, Kolb M. Why do patients get idiopathic pulmonary fibrosis? Current concepts in the pathogenesis of pulmonary fibrosis. BMC Med 2015; 13: 176.

3 Schroedl CJ, Yount SE, Szmuilowicz E, et al. A qualitative study of unmet healthcare needs in chronic obstructive pulmonary disease. A potential role for specialist palliative care? Ann Am Thorac Soc 2014; 11: 1433-1438.

4 Mura M, Porretta MA, Bargagli E, et al. Predicting survival in newly diagnosed idiopathic pulmonary fibrosis: a 3-year prospective study. Eur Respir J 2012; 40: 101-109.

5 Albera C. Challenges in idiopathic pulmonary fibrosis trials: the point on end-points. Eur Respir Rev 2011; 20 195-200.

6 Gordon JK, Domsic RT. Clinical trial design issues in systemic sclerosis: an update. Curr Rheumatol Rep 2016; 18: 38.

7 Layton AM, Armstrong HF, Kim HP, et al. Cardiopulmonary exercise factors predict survival in patients with advanced interstitial lung disease referred for lung transplantation. Respir Med 2017; 126: 59-67.

8 Sue DY, Wasserman K. Impact of integrative cardiopulmonary exercise testing on clinical decision making. Chest 1991; 99: 981-992.

9 Palange $\mathrm{P}$, Ward SA, Carlsen $\mathrm{KH}$, et al. Recommendations on the use of exercise testing in clinical practice. Eur Respir J 2007; 29: 185-209. 
10 Ferrazza AM, Martolini D, Valli G, et al. Cardiopulmonary exercise testing in the functional and prognostic evaluation of patients with pulmonary diseases. Respiration 2009; 77: 3-17.

11 Arena R, Sietsema KE. Cardiopulmonary exercise testing in the clinical evaluation of patients with heart and lung disease. Circulation 2011; 123: 668-680.

12 Bonini M, Fiorenzano G. Exertional dyspnoea in interstitial lung diseases: the clinical utility of cardiopulmonary exercise testing. Eur Respir Rev 2017; 26: 160099.

13 American Thoracic Society, American College of Chest Physicians. ATS/ACCP Statement on Cardiopulmonary Exercise Testing. Am J Respir Crit Care Med 2003; 167: 211-277.

14 Shamseer L, Moher D, Clarke M, et al. Preferred reporting items for systematic review and meta-analysis protocols (PRISMA-P) 2015: elaboration and explanation. BMJ 2015; 350: g7647.

15 Altman DG, Decks JJ, Clarke M, et al. The quality of systematic reviews. High quality reporting of both randomised trials and systematic reviews should be priority. BMJ 2000; 321: 297-299.

16 Cohen J. Weighted kappa: nominal scale agreement with provision for scaled disagreement or partial credit. Psychol Bull 1968; 70: 213-220.

17 Huguet A, Hayden JA, Stinson J, et al. Judging the quality of evidence in reviews of prognostic factor research: adapting the GRADE framework. Syst Rev 2013; 2: 71.

18 Gläser S, Obst A, Koch B, et al. Pulmonary hypertension in patients with idiopathic pulmonary fibrosis - the predictive value of exercise capacity and gas exchange efficiency. PLoS One 2013; 8: e65643.

19 Kawut SM, O'Shea MK, Bartels MN, et al. Exercise testing determines survival in patients with diffuse parenchymal lung disease evaluated for lung transplantation. Respir Med 2005; 99: 1431-1439.

20 van der Plas MN, van Kan C, Blumenthal J, et al. Pulmonary vascular limitation to exercise and survival in idiopathic pulmonary fibrosis. Respirology 2014; 19: 269-275.

21 Miki K, Maekura R, Hiraga T, et al. Impairments and prognostic factors for survival in patients with idiopathic pulmonary fibrosis. Respir Med 2003; 97: 482-490.

22 Wallaert B, Guetta A, Wemeau-Stervinou L, et al. [Prognostic value of clinical exercise testing in idiopathic pulmonary fibrosis]. Rev Mal Respir 2011; 28: 290-296.

23 King TE, Tooze JA, Schwarz MI, et al. Predicting survival in idiopathic pulmonary fibrosis: scoring system and survival model. Am J Respir Crit Care Med 2001; 164: 1171-1181.

24 Fell CD, Liu LX, Motika C, et al. The prognostic value of cardiopulmonary exercise testing in idiopathic pulmonary fibrosis. Am J Respir Crit Care Med 2009; 179: 402-407.

25 Kollert F, Geck B, Suchy R, et al. The impact of gas exchange measurement during exercise in pulmonary sarcoidosis. Respir Med 2011; 105: 122-129.

26 Lopes AJ, Menezes SL, Dias CM, et al. Cardiopulmonary exercise testing variables as predictors of long-term outcome in thoracic sarcoidosis. Braz J Med Biol Res 2012; 45: 256-263.

27 Swigris JJ, Zhou X, Wamboldt FS, et al. Exercise peripheral oxygen saturation $\left(\mathrm{SpO}_{2}\right)$ accurately reflects arterial oxygen saturation $\left(\mathrm{SaO}_{2}\right)$ and predicts mortality in systemic sclerosis. Thorax 2009; 64: 626-630.

28 Triantafillidou C, Manali E, Lyberopoulos P, et al. The role of cardiopulmonary exercise test in IPF prognosis. Pulm Med 2013; 2013: 514817.

29 Vainshelboim B, Oliveira J, Fox BD, et al. The prognostic role of ventilatory inefficiency and exercise capacity in idiopathic pulmonary fibrosis. Respir Care 2016; 61: 1100-1109.

30 American Thoracic Society, European Respiratory Society. American Thoracic Society/European Respiratory Society International Multidisciplinary Consensus Classification of the Idiopathic Interstitial Pneumonias. Am J Respir Crit Care Med 2002; 165: 277-304.

31 Raghu G, Collard HR, Egan JJ, et al. An Official ATS/ERS/JRS/ALAT Statement: Idiopathic Pulmonary Fibrosis: Evidence-based Guidelines for Diagnosis and Management. Am J Respir Crit Care Med 2011; 183: 788-824.

32 Scadding JG. Prognosis of intrathoracic sarcoidosis in England. A review of 136 cases after five years' observation. Br Med J 1961; 2: 1165-1172.

33 Massi AT. Subcommittee for Scleroderma Criteria of the American Rheumatism Association Diagnostic and Therapeutic Criteria Committee. Preliminary criteria for the classification of systemic sclerosis (scleroderma) Arthritis Rheum 1980; 23: 581-590.

34 Poormoghim H, Lucas M, Fertig N, et al. Systemic sclerosis sine scleroderma: demographic, clinical, and serologic features and survival in forty-eight patients. Arthritis Rheum 2000; 43: 444-451.

35 Gibbons RJ, Balady GJ, Beasley JW, et al. ACC/AHA Guidelines for Exercise Testing. A report of the American College of Cardiology/American Heart Association Task Force on Practice Guidelines (Committee on Exercise Testing). J Am Coll Cardiol 1997; 30: 260-311.

36 McArdle WD, Katch FI, Pechar GS. Comparison of continuous and discontinuous treadmill and bicycle tests for max Vo2. Med Sci Sports 1973; 5: 156-160.

37 Bujang MA, Sa'at N, Sidik TMITAB. Determination of minimum sample size requirement for multiple linear regression and analysis of covariance based on experimental and non-experimental studies. Epidemiol Biostat Public Health 2017; 14: e12117-1-9.

38 Whittingham MJ, Stephens PA, Bradbury RB, et al. Why do we still use stepwise modelling in ecology and behaviour? J Anim Ecol 2006; 75: 1182-1189.

39 Derksen S, Keselman HJ. Backward, forward and stepwise automated subset selection algorithms: frequency of obtaining authentic and noise variables. Br J Math Stat Psychol 1992; 45: 265-282.

40 Chatterjee S, Hadi AS, Bertram P. Regression Analysis by Example (Series in Probability and Statistics). 3rd Edn Hoboken, Wiley, 2000.

41 Nathan SD, Meyer KC. IPF clinical trial design and endpoints. Curr Opin Pulm Med 2014; 20: 463-471.

42 Boutou AK, Pitsiou GG, Trigonis I, et al. Exercise capacity in idiopathic pulmonary fibrosis: the effect of pulmonary hypertension. Respirology 2011; 16: 451-458.

43 Collard HR, King TE, Bartelson BB, et al. Changes in clinical and physiologic variables predict survival in idiopathic pulmonary fibrosis. Am J Respir Crit Care Med 2003; 168: 538-542.

44 Hemingway H, Riley RD, Altman DG. Ten steps towards improving prognosis research. BMJ 2009; 339: b4184. 